\title{
Reduction of droplet-size dispersion in parallel flow-focusing microdevices using a passive method
}

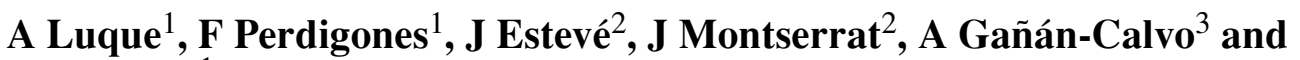 \\ J M Quero ${ }^{1}$ \\ ${ }^{1}$ Department of Electronic Engineering, University of Seville, Spain \\ ${ }^{2}$ Centro Nacional de Microelectronica (CNM-IMB), Spain \\ ${ }^{3}$ Department of Aerospace Engineering and Fluid Mechanics, University of Seville, Spain \\ E-mail: aluque@gte.esi.us.es
}

Received 28 October 2008, in final form 4 March 2009

Published 26 March 2009

Online at stacks.iop.org/JMM/19/045029

\begin{abstract}
Flow-focusing devices can be used to produce microparticles at low cost, with the added advantage of low dispersion in the size of the generated particles. However, when multiple parallel devices are used with common inputs to massively produce the microparticles, the overall production is polydisperse, usually due to differences in flow rates of the focused fluid through each single device. The solution to uniformize this flow rate can involve active, movable devices that would add complexity and cost to the system. A simpler solution is to add distribution and equalization channels that drive focused fluid to the inputs. Experimental results show that this method can reduce the total dispersion, and render the multiple device close to monodispersion.
\end{abstract}

\section{Introduction}

Production of micrometer-sized liquid droplets or solid particles has found applications in diverse fields, among which printing has always been considered the traditional one [1].

Recently, sectors such as biomedical, chemical or pharmaceutical have turned attention to MEMS as the adequate tools for producing the required particles with the appropriate requirements: size, size dispersion and production rate [2]. For example, when producing drugs intended for inhalation, they can be targeted to specific areas of the respiratory track by aiming to a particular deposition mechanism, which is determined by droplet size. It is essential to be able to precisely control size of the produced droplets, as well as ensuring that the random dispersion around this size is as low as possible $[3,4]$.

When the purpose is to generate solid microparticles of a given size, one of the most common methods is to create droplets of a solution of the desired material, and then let them dry out until only the solid remains. Different methods are used to ensure that droplets do not deform or stick together in the drying process.
Many different methods exist to produce droplets at the microscale, each one with its own set of advantages and disadvantages. To name a few, acoustic waves [5], heaters [6], piezoelectric actuators [7] and micronozzles [8, 9] have been successfully employed.

The technique known as flow focusing is also widely used to create droplets of a small size [10-14]. It is based on mechanical effects only, and its robustness and yield make it suitable for a vast array of applications. One of its main advantages is the low dispersion in droplet size that is easily achieved. In addition, droplet size can be controlled by changing flow rate or pressure of one or both of the fluids involved. Different fabrication processes have been designed to construct flow-focusing devices [15, 16]. Traditionally, emphasis has been put on the low cost or high size control, and this has led to the development of bidimensional flowfocusing devices [17] or three-dimensional focusing devices that are not integrable in large arrays [18-20]. If the final aim is the massive production, then devices whose geometry allows them to be integrated in a large number are required.

In this paper, a contribution toward the development of massively parallel flow-focusing devices is presented. It will 


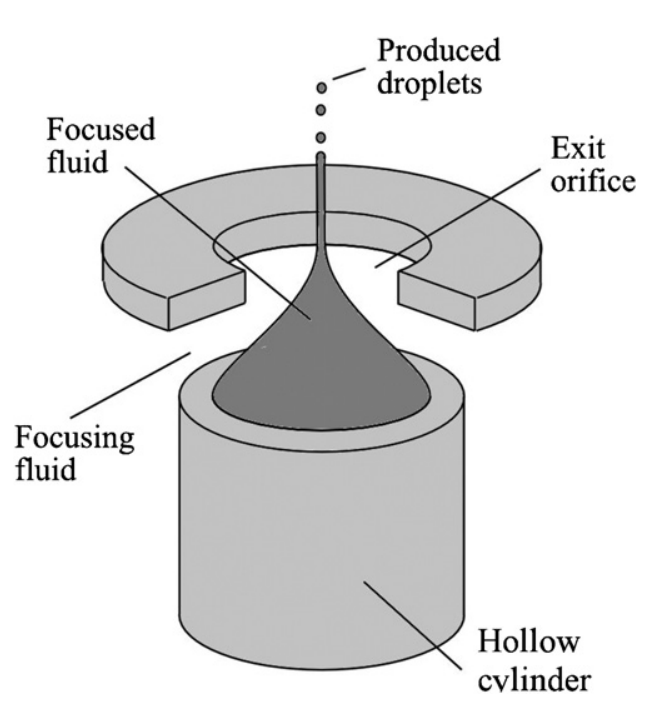

Figure 1. Structure of a flow-focusing device, showing focused and focusing fluid.

be shown how the low dispersion typical of flow-focusing devices is turned into polydispersion when multiple devices are fed from the same source. The goal of the research presented here was to find a simple and inexpensive way to return this polydispersion again to monodispersion while maintaining all the other features of the technique.

The remainder of this paper is organized as follows. Section 2 will describe how the flow-focusing technique works, how microdevices can be fabricated in silicon using microfabrication technologies, and the dispersion obtained when multiple devices are used in parallel to produce droplets. In section 3 , the proposed solution will be shown, the results obtained in experimental tests will be discussed and compared with those obtained previously. Finally, in section 4 some conclusions will be highlighted.

\section{Parallel flow-focusing devices}

In this section, the flow-focusing technique (in particular, three-dimensional flow focusing) will be presented, showing actual microdevices that have been successfully used to produce droplets or other microparticles [19, 21, 22]. As stated in introduction, the integration of a large number of flow-focusing devices in the same chip can lead to massive production of microparticles at a low cost, so the design and fabrication process presented here will be oriented toward this final objective.

\subsection{Design and fabrication}

The basis of the flow-focusing technique can be seen in figure 1. A three-dimensional flow-focusing device is composed by a hollow cylinder, or pipe, whose axis is aligned with an exit orifice located on a plate. A stream of focused fluid flows through the cylinder, and, before coming out through the exit orifice, is focused by a second fluid, called focusing fluid. Both fluids need to be immiscible between them. Depending on the force exerted by the focusing fluid over the focused one, the final stream diameter will vary. This stream breaks into droplets following Rayleigh's law of capillary breakup, producing droplets of the desired diameter. In order for this to happen, the focused fluid must be a liquid and the focusing one a gas. When focused fluid is a gas and focusing fluid is a liquid, bubbles are produced, and if both fluids are liquid, emulsions of a liquid inside another are created. In all cases, the flow-focusing principle is the same.

It is known $[23,24]$ that the diameter of the generated focused stream $d_{j}$ can be expressed as

$$
d_{j}=\left(\frac{8 \rho_{d}}{\pi^{2} \Delta p_{t}}\right)^{1 / 4} Q_{d}^{1 / 2}+\frac{\sigma}{2 \Delta p_{t}},
$$

where $\rho_{d}$ is the density of the focused fluid, $\Delta p_{t}$ is the pressure at which the focusing fluid is supplied, $Q_{d}$ is the flow rate of the focused fluid and $\sigma$ is the surface tension between both fluids. The droplet size depends on the Weber number (ratio between inertial and interfacial forces), given by

$$
\mathrm{We}=\frac{\Delta p_{t} d_{j}}{\sigma}
$$

If it is assumed that the stream diameter is constant after exiting the device, and it breaks into droplets following Weber and Rayleigh's predictions, it can be deduced [25] that for low Weber numbers $(1<\mathrm{W}<20)$, droplet diameter is given by

$$
d_{d}=(3 \pi / \sqrt{2})^{1 / 3}(1+Z)^{1 / 6} d_{j}
$$

where $Z=\left(3 \mu_{d}+\mu_{t}\right) /\left(d_{j} \sigma \rho_{d}\right)^{1 / 2}$, and $\mu_{d}$ and $\mu_{t}$ are the viscosities of the focused and focusing fluids, respectively.

When $Z \ll 1$, the diameter of the produced droplets $d_{d}$ can be expressed as

$$
d_{d} \simeq 1.89 d_{j} .
$$

One of the most important features of flow focusing is that geometry of the device does not directly influence the size of the generated droplets $[11,24]$, as it can be deduced from the fact that geometrical parameters do not appear in (1). Therefore, fabrication tolerances in the dimensions do not affect the final result, as long as these dimensions still fall within the operating regime. However, dimensions can indirectly influence the results in some situations. For example, if focused fluid is fed at a constant pressure, then the cylinder inner diameter will have an effect over $Q_{d}$ and consequently over $d_{d}$ and $d_{j}$.

When there is need to improve the throughput and massively produce the particles, multiple flow-focusing devices can be made to work in parallel, sharing common inputs for focused and focusing fluids. If the fabrication process allows devices to be put in a bidimensional array, a high level of integration can be achieved. The final chip will have several exit orifices, and two inlet ports: one for the focusing and one for the focused fluid.

Figure 2 shows the simplest parallel device, with two exits. To be able to achieve this integration, the direction of the issued streams must be perpendicular to the substrate [22]. The open space inside the device is designed to behave as a stagnation chamber. The input orifice for the focusing fluid has an area much larger than the sum of all the outputs, guaranteeing the pressure inside $\Delta p_{t}$ will not depend on flow rate through each 


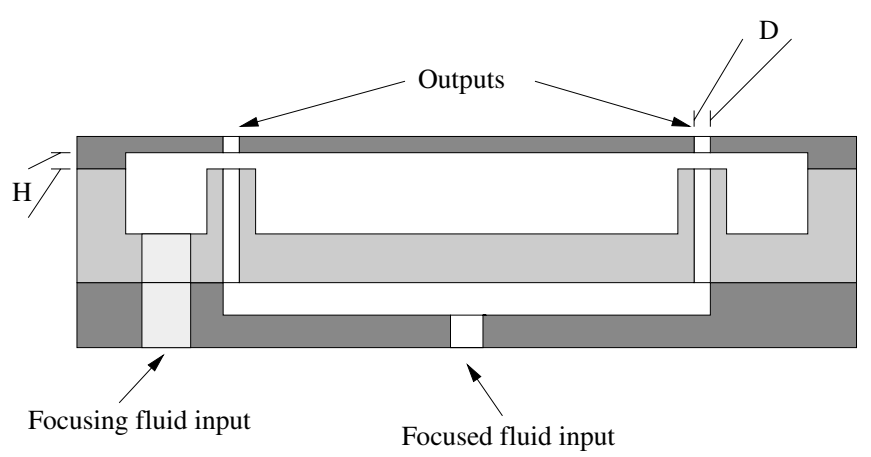

Figure 2. Distribution of two flow-focusing devices in the same die (cross-section).

(a)

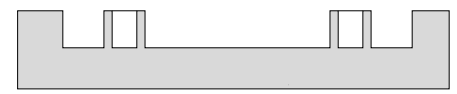

(b)

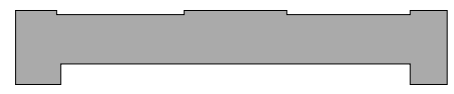

(c)

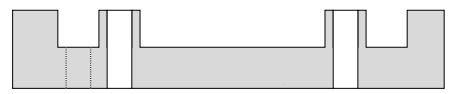

(d)

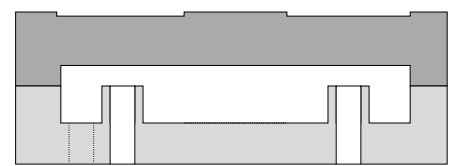

(e)

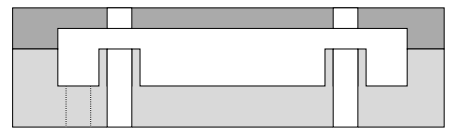

$(f)$

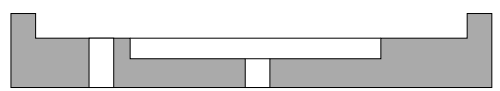

Figure 3. Fabrication process.

exit, and will be the same for each individual device. The input for the focused fluid is connected to a channel network that distributes the flow to all individual devices.

Fabrication processes have been previously presented in the available literature, both for constructing bidimensional focusing devices [26] and three-dimensional ones [27], but the purpose here is to make three-dimensional focusing devices integrated in a bidimensional array. The process used in this work is based on that presented in [22]. Two silicon wafers are used, which are separately micromachined and then bonded together to form the final structure.

The process starts by performing photolithography on the wafer that will form the bottom part of the finished device. The thickness of this wafer is $500 \mu \mathrm{m}$. The area that the devices will take up is defined, and then reactive ion etching (RIE) is used to remove material and create the chambers where the focusing fluid will be distributed around the stream of focused fluid (figure 3(a)). The etched depth is $180 \mu \mathrm{m}$, and the etching is made using a pulsed process of $\mathrm{SF}_{6}$ and $\mathrm{C}_{4}$ $\mathrm{F}_{8}$ (Bosch process) at ambient temperature. The hard mask that is used in all DRIE steps throughout the process is made of aluminum.

After this, a second wafer, $300 \mu \mathrm{m}$ thick, is machined from its bottom side (figure $3(b)$ ). The depth of this etching defines the dimension $H$ in figure 2, and the etching is performed over the same area where the focusing fluid delivery network was placed in step (a). In this work, $H$ was chosen to be $60 \mu \mathrm{m}$. The same pattern is marked on the opposite side of the wafer, so that the position of the features can be known when both wafers are bonded together.

In a third step (figure 3(c)) the bottom wafer is etched from the bottom side, in order to create the input orifices for focusing fluid. The alignment of the mask used in this etching with the previous openings is made using back-side alignment.

Both wafers are then bonded together using silicon fusion bonding. Wafer surfaces are activated using oxygen plasma, and then wafers are put at $1100{ }^{\circ} \mathrm{C}$, first in an oxidant environment, and in a second stage in an inert ambient.

In step (e), exit orifices are opened. The photolithography in this step makes use of back-side alignment and aligns the new mask against the input orifices that were etched in the bottom side of the bottom wafer in step (c). This way, the exit orifice is placed in front of the cylinder from which the focused fluid will come. The diameter $D$ of the exit orifice is $100 \mu \mathrm{m}$.

Finally (figure $3(f)$ ) a holding and distribution device made of steel is used to place the inlet ports and the distribution network for the focused fluid. This distribution network is composed by channels through which the focused fluid flows from a common input to each one of the inputs of the hollow cylinders.

In figure $4(a)$ the fabricated cylinder can be seen after the step $(a)$ of the fabrication process and figure $4(b)$ shows a cross-section of the final silicon device. In this figure, the cut cylinder is shown, as well as the channel through which the focused fluid flows and part of the chamber for the focusing fluid.

\subsection{Experimental results}

After the fabrication of two-exits parallel flow-focusing devices, experimental tests were carried out to measure the average size and dispersion of the generated droplets. A particle size analyzer by laser diffraction from Sympatec $\mathrm{GmbH}$, Germany, was used for this task.

Figure 5 shows the average droplet size for each one of the exit orifices, $\mathrm{A}$ and $\mathrm{B}$, as a function of the focused flow rate $Q_{d}$, which varied from 7 to $50 \mathrm{~mL} \mathrm{~h}^{-1}$. Pressure for focusing fluid was kept constant at 215 mbar. In this case, the distribution network for the focused fluid consisted solely of a chamber from where both cylinders were fed. In the figure, theoretical value is obtained by taking the total flow rate fed to both orifices, dividing it by half, and using (1) and (4) to obtain the expected droplet size.

It can be seen that there is a certain difference with respect to the theoretical expected value. According to (1), this is due to different focused flow rates coming through each cylinder and exit orifice. If both share a common input 


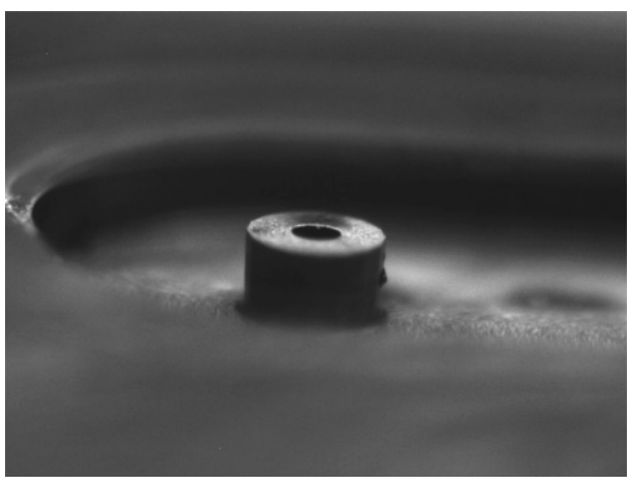

(a)

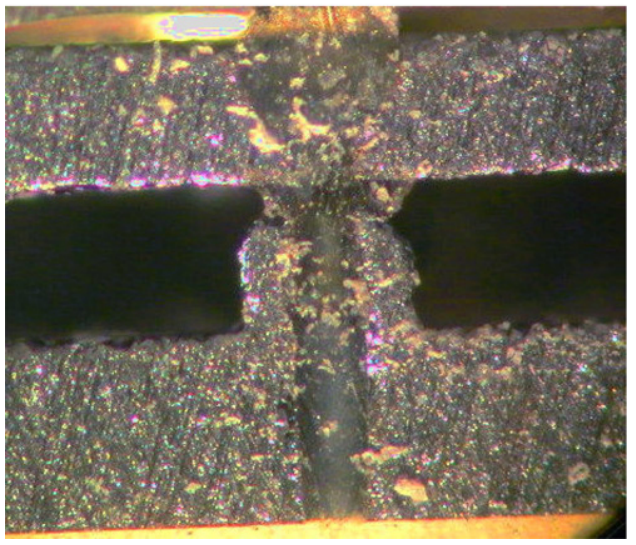

(b)

Figure 4. (a) View of the flow-focusing cylinder made of silicon. The stagnation chamber can also be seen in the picture.

(b) Cross-section of the fabricated device.

(This figure is in colour only in the electronic version)

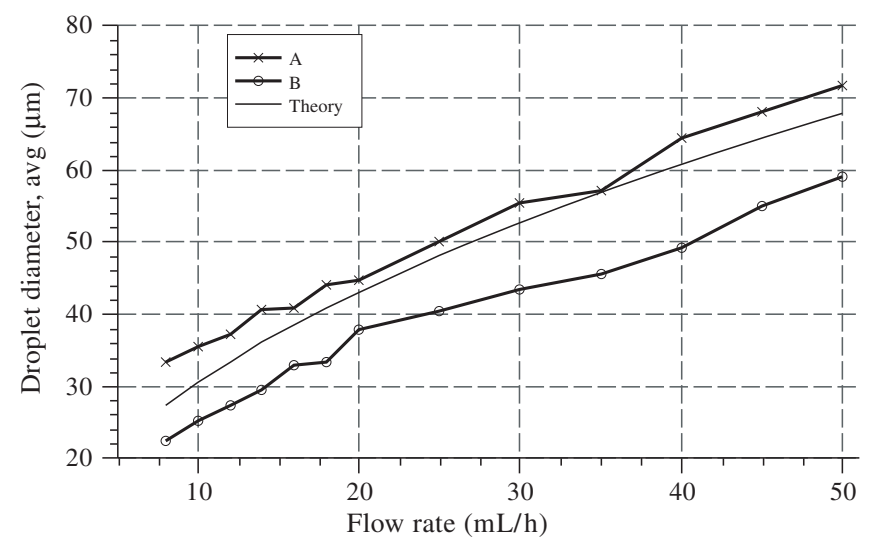

Figure 5. Measured average droplet diameter as a function of liquid flow rate for both outputs.

kept at a given pressure, the difference in flow rate comes from some geometrical variation in cylinder inner diameter or length caused by fabrication errors or tolerances. In particular, a small variation in the cylinder inner diameter caused by misalignment or operating differences during the DRIE process can have a considerable effect over the flow rate.

In addition to the average size, the dispersion in size can also be measured. Figure 6 shows the distribution

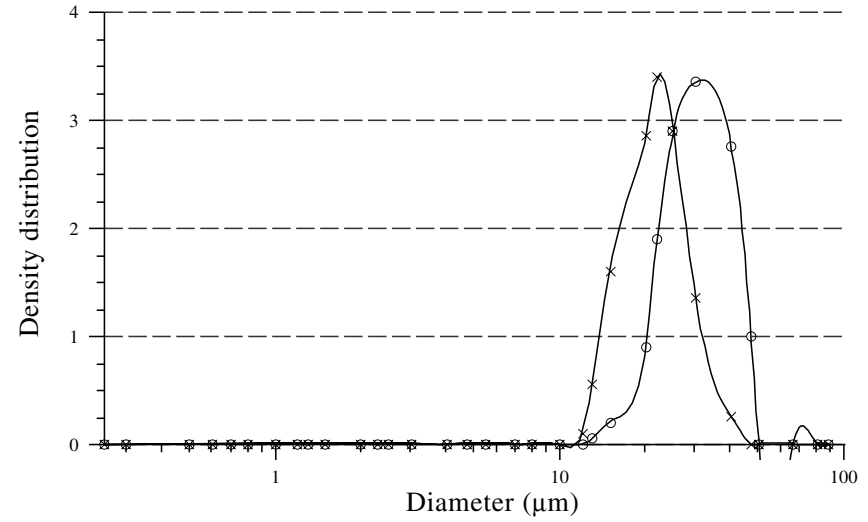

Figure 6. Density distribution of droplet diameter for both outputs at $Q_{d}=8 \mathrm{mLh}^{-1}$.

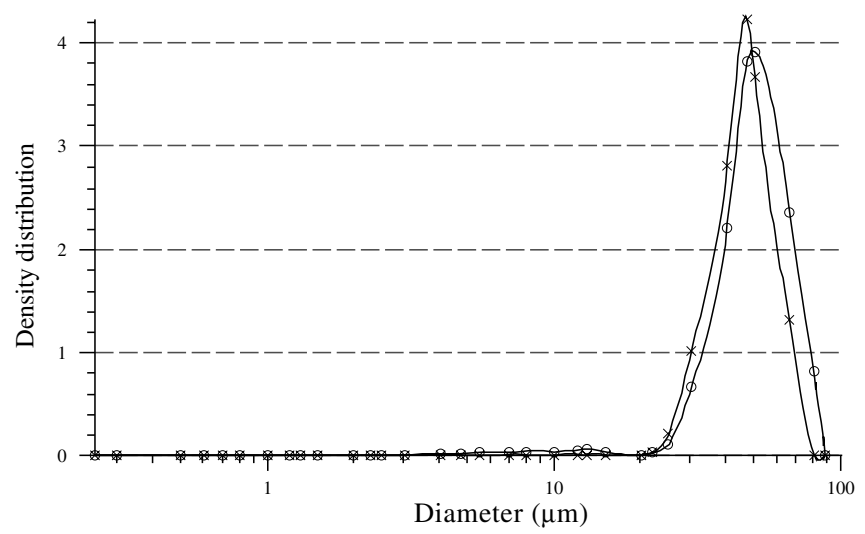

Figure 7. Density distribution of droplet diameter for both outputs at $Q_{d}=30 \mathrm{~mL} \mathrm{~h}^{-1}$.

density on the diameter of droplets produced at each orifice at $Q_{d}=8 \mathrm{~mL} \mathrm{~h}^{-1}$. A certain dispersion exists in each orifice around their average values. The observed behavior is that of log-normal distributions, with the mass median diameter being the one depicted in figure 5 .

Geometric standard deviations (GSD) in droplet size for each exit orifice are respectively 1.27 and 1.31. But when both orifices are considered together the total GSD grows up to 1.38 , clearly showing the existent polydispersion in the parallel device. Ideally, GSD should be close to 1, while values between 1.2 and 1.3 are generally considered as monodispersion.

Figure 7 shows the density distribution of droplet size for a focused fluid flow rate of $30 \mathrm{~mL} \mathrm{~h}^{-1}$. It can be seen how the global behavior is still polydisperse, but this is much less visible than in the previous case. For larger flow rates, mean droplet sizes tend to be more similar and the overall operation is closer to monodispersion. Nevertheless, as focused flow rate is one of the main parameters affecting droplet size, it is not always possible to use large flow rates to minimize polydispersion, especially when the aim is to produce small droplets (around $10 \mu \mathrm{m}$ or less). In these cases, an effective, size-independent method to approach monodispersion becomes necessary. 


\section{Improvement of monodispersion}

\subsection{Proposed solution}

When using parallel chips in which all single devices are intended to produce droplets of the same size, it is important to guarantee that all devices are fed with the same pressure for focusing fluid and the same flow rate for the focused one.

For the focusing gas, this is usually accomplished by placing a stagnation chamber common to all devices, as explained before. A stagnation chamber is large enough so that its pressure does not vary when a flow of air is coming out from it. An external pressure pump is used to keep the pressure constant at the desired value.

As stated above, if focused fluid is provided at a given pressure, tolerances and errors that arise in the fabrication processes at the microscale make each conducting channel and cylinder slightly different from each other, which in turn makes each flow rate and droplet size different. This is the explanation of the polydispersion observed in figures 6 and 7 .

One alternative is to feed the chip at a given and constant flow rate by using a syringe pump, and then a flow divider would be used that would distribute flow between all inputs. But again tolerances and errors would make flows different.

A possible solution then could be to try to reduce the fabrication tolerances and improve the fabrication process, but this approach is not practical when dealing with very small devices and trying to achieve very low dispersion. It is simply not possible to make the process so reliable (with errors below the micron) at a reasonable cost.

Other techniques can be used to uniformize flow rates between single devices, such as a feedback loop that would measure flows and would adjust a variable fluidic resistance, so that at the end all of them are essentially equal. The active fluidic component that changes its resistance as a function of an external signal would need to be integrated in the silicon chips. Apart from the inherent difficulty of constructing such a feedback loop and fluidic components at a small scale, the cost would again be very high, and probably not worth the effort.

The proposed solution to this problem is to include passive fluidic components which are inexpensive to build and easily integrable with the devices. A constant fluidic resistance can be added to each device upstream of the cylinder. The added resistance will be larger than the total resistance in the cylinder, making the differences between cylinders negligible $[28,29]$. This is easily accomplished by modifying the distribution piece that holds the device and adjusting length and width of the distribution channels until their fluidic resistance is adequate. The distribution network then becomes a distribution and equalization network. The fluidic resistance serves the function of isolating individual devices from each other, so that from the common focused fluid input, all of them present the same resistance to flow, and thus the flow rates will be equal.

This approach has been experimentally tested, and section 3.2 will describe the measurements that clearly show an improvement toward monodispersion.
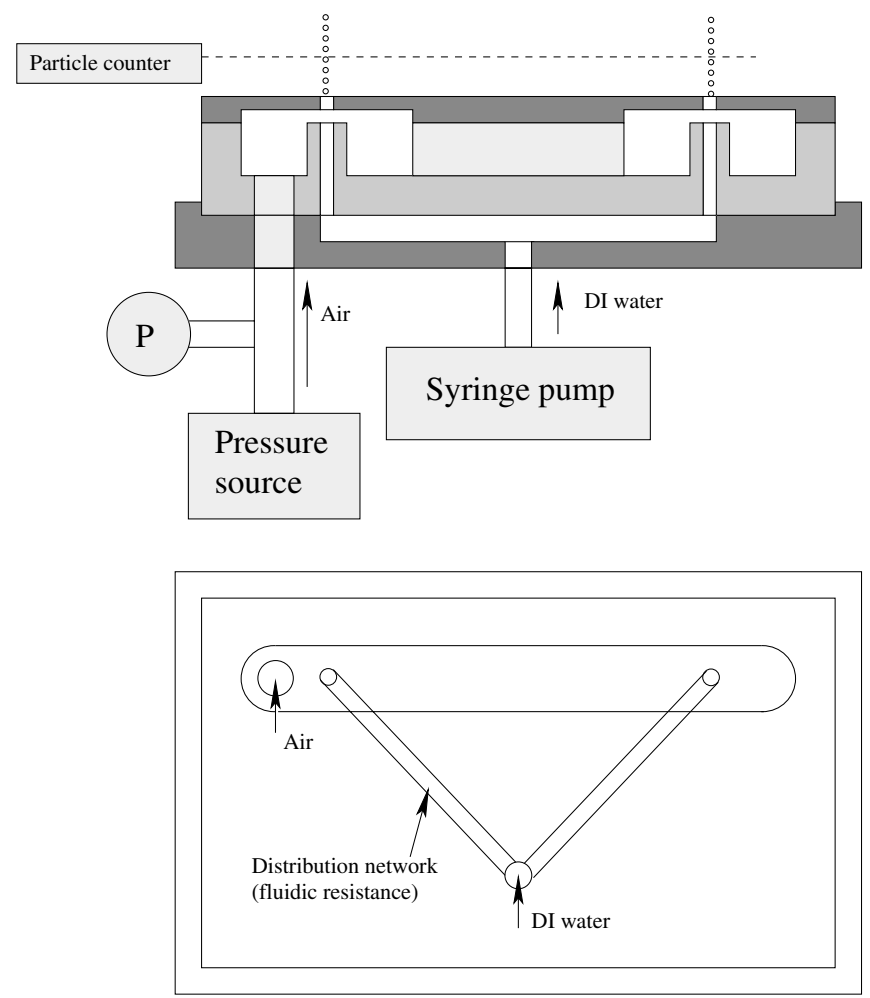

Figure 8. Experimental setup aimed at measuring average droplet size and dispersion and top view of the distribution network and fluidic inputs.

\subsection{Experimental results}

The set of experiments was performed on a die which contained two flow-focusing devices with the same designed dimensions. Both exits were $6 \mathrm{~mm}$ apart, and the total size of the die was $10 \times 10 \mathrm{~mm}$. The purpose of the experiments was to measure the improvement in monodispersion, if any. This was done by measuring average droplet size in each exit, and global GSD in the die.

The experimental setup can be seen in figure 8. Focused fluid was DI water, and was fed to the parallel device using a syringe pump. Focusing fluid was air and was entered at a constant pressure, measured by a pressure gauge. Particle size was again measured using a laser particle analyzer.

Figure 8 also shows a top view of the multiple device. The distribution network that carries the focused fluid (DI water) from the common input to each one of the individual flow-focusing devices can be seen.

Two different distribution networks for focused fluid were used: one with channels $40 \mathrm{~mm}$ long and square section $100 \mu \mathrm{m}$ wide, and the other with $32 \mathrm{~mm}$ long channels with square section $150 \mu \mathrm{m}$ wide. Fluidic resistance varies with $L / d_{e}^{4}$, being $L$ channel length, and $d_{e}$ the equivalent diameter, so the resistance of the first network is significantly larger that that of the second one. Distribution channels had a common input from where the focused fluid (DI water) was fed using a syringe pump. During all experiments, pressure for the focusing fluid was kept constant at $215 \mathrm{mbar}$ and flow rate for focused fluid was varied. 


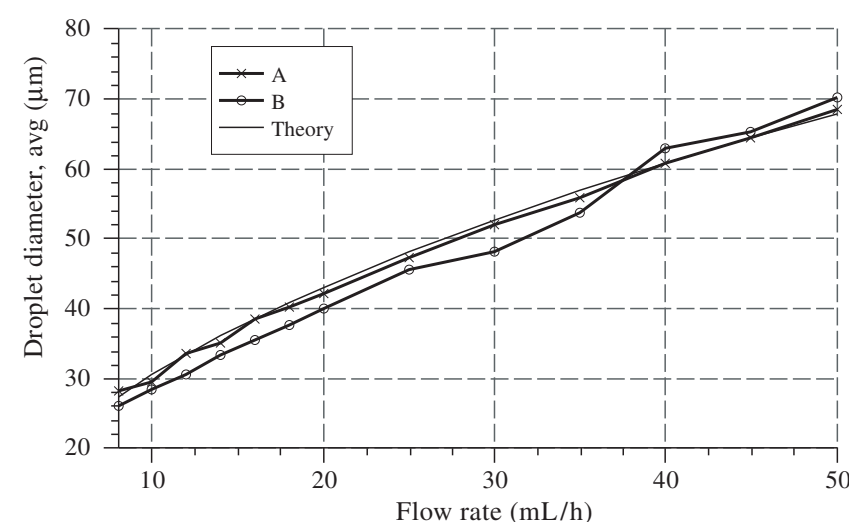

(a)

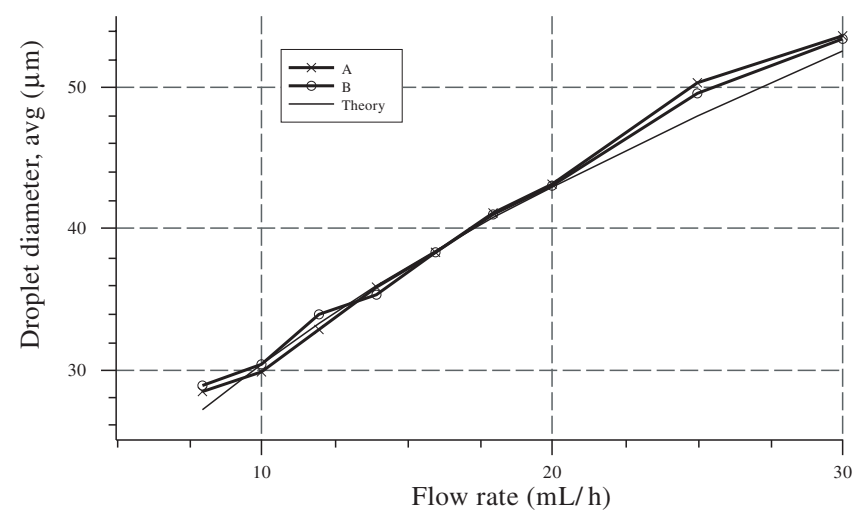

(b)

Figure 9. Measured average droplet diameter as a function of liquid flow rate for both outputs with different distribution channels (a) $32 \mathrm{~mm} 150 \mu \mathrm{m}$ (b) $40 \mathrm{~mm} 100 \mu \mathrm{m}$.

Figures $9(a)$ and $(b)$ show the average droplet size in each orifice as a function of the focused flow rate. In the ideal case, droplet size from both exits would be coincident at the theoretical value depicted in the figure. If this figure is compared with figure 5, visible improvements can be noted and, furthermore, these improvements are more abrupt when fluidic resistance in the distribution network is larger (figure $9(b)$ ).

The influence of the total resistance to flow in the distribution network can be seen in figure 10. In the horizontal axis, resistance to flow (calculated as $L / d_{e}^{4}$ ) is indicated. The figure shows that when distribution channels present a larger fluidic resistance, droplet diameters at both exits tend to the theoretical value (shown as an horizontal solid line in the figure). With this figure, an estimation of the length and width of the needed channels from the allowed deviation from theoretical value can be made.

Probability density distributions for the generated diameters are shown in figure 11. Measured points are marked in the plots.

In figure 11(a), diameter density distribution is shown for both outputs when no distribution network was added. This is the same image as figure 6. This figure can be compared with figure 11(b), which shows the size distribution when the distribution network ( $32 \mathrm{~mm}, 150 \mu \mathrm{m}$ channels) is added. It can be clearly seen how the global average is the same, while

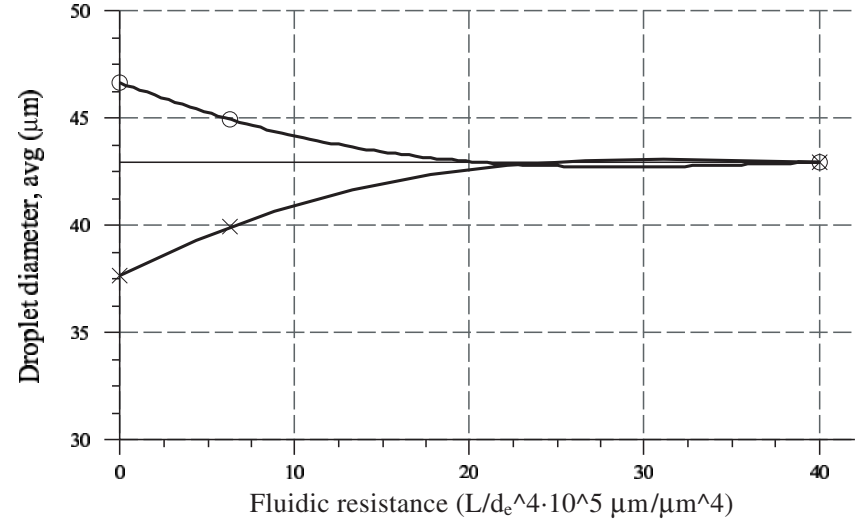

Figure 10. Measured average diameter for droplet produced by a multiple device with two outputs, as a function of the added resistance to flow for a liquid flow rate $Q_{d}=20 \mathrm{~mL} \mathrm{~h}^{-1}$.

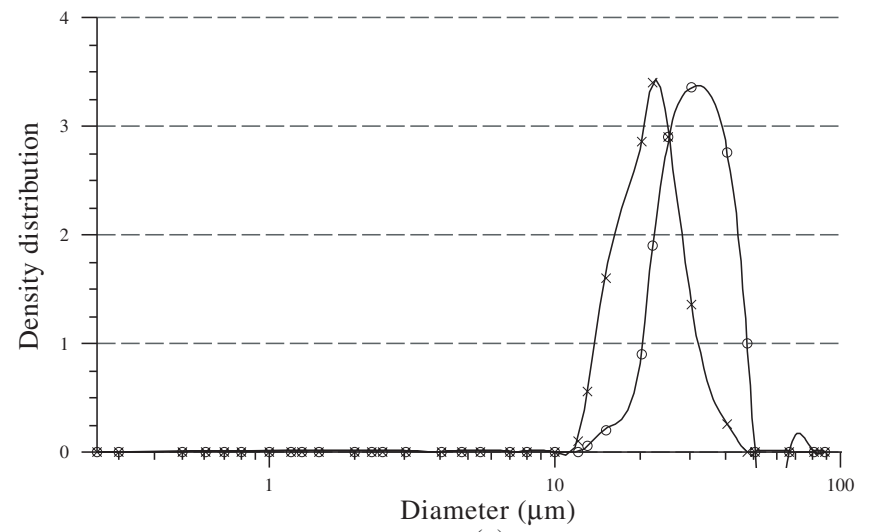

(a)

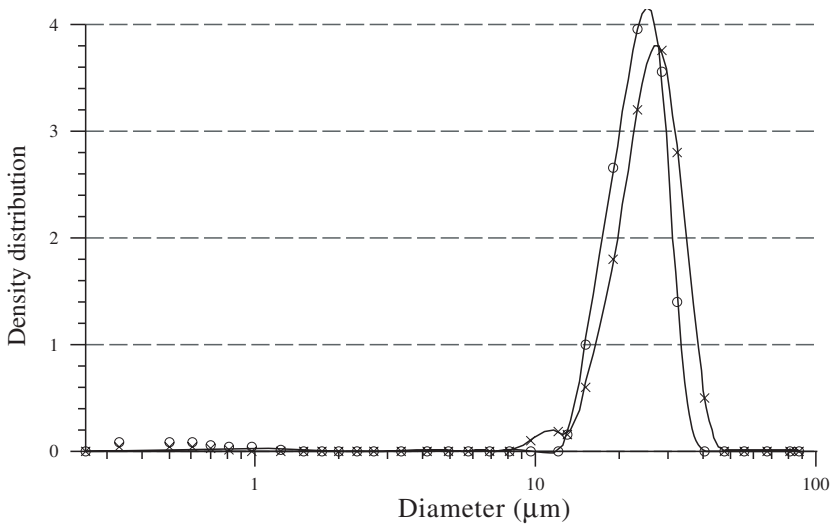

(b)

Figure 11. Density distribution of droplet diameter for both outputs, at $Q_{d}=8 \mathrm{~mL} \mathrm{~h}^{-1},(a)$ without a distribution network, $(b)$ with distribution network.

the dispersion is significantly lower. In the case of figure 11(a), GSDs for each exit have the values 1.27 and 1.32, which shows that the individual devices are approximately monodisperse. But the GSD of the two devices considered at the same time is 1.36 , which is far away from ideal monodispersion. When the distribution network is added, the global GSD goes down to 1.26 , which signals that monodispersion has been reached again. 


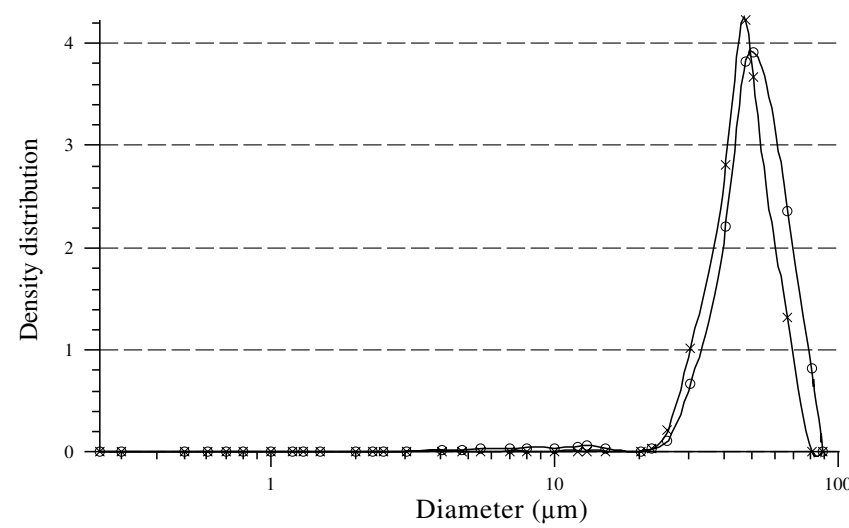

(a)

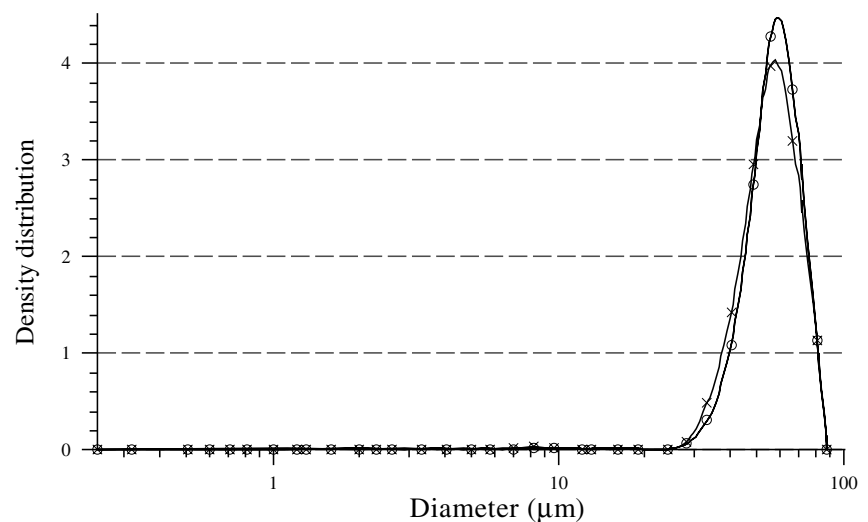

(b)

Figure 12. Density distribution of droplet diameter for both outputs, at $Q_{d}=30 \mathrm{~mL} \mathrm{~h}^{-1},(a)$ without distribution network, $(b)$ with distribution network.

A different set of experiments is shown in figure 12. They represent the results when the focused flow rate is $15 \mathrm{~mL} \mathrm{~h}^{-1}$. Again, figure 12(a) corresponds to the experiment without distribution network, and figure $12(b)$ with it. In the first figure, individual GSDs take the values 1.22 and 1.37 , while the global GSD is 1.35 . When the channels are added, global GSD takes the value 1.25 , again in a monodispersion regime.

\section{Conclusions}

The proposed method of including a distribution network for the focused fluid effectively reduces the global dispersion of a multiple flow-focusing device, making the overall droplet production very close to monodispersion, or even monodisperse. This solution allows the fabrication of massive droplet generation devices using the flow-focusing technique and guaranteeing little dispersion around the desired droplet size. As the method consists in adding only passive components to the fluidic circuit, it can be integrated with the fabrication process in an inexpensive way.

The fluidic resistance added between the common input and the individual devices isolates each flow-focusing device from the rest. As long as each exit is placed far enough from each other, an arbitrary number of individual devices can be placed in the same chip. A star-shaped configuration of the distribution network can always be used to distribute the focused fluid flow.
The addition of the distribution and equalization network channels increases the power consumption, but in all practical applications the power needed to impulse the liquid focused fluid is negligible when compared to that employed in the focusing gas.

The study and experimental results presented here have focused on the static analysis of the behavior of flow-focusing devices, and tried to solve the variation on droplet size when operating in steady state. The dynamic interaction among devices was not the subject of this research. Such a study on the dynamic behavior and the potential instabilities that could arise between streams or droplets when multiple devices are placed close to each other would be necessary to advance further in the design of massively parallel microdroplet production systems.

\section{References}

[1] Heinzl J and Hertz C H 1985 Ink-jet printing Adv. Electron. Electron. Phys. 65 91-171

[2] Lee E R 2003 Microdrop Generation (Boca Raton, FL: CRC Press)

[3] Newman S P, Pellow P G D and Clarke S W 1986 Droplet size distributions of nebulised aerosols for inhalation therapy Clin. Phys. Physiol. Meas. 7 139-46

[4] Gomez A 2002 The electrospray and its application to targeted drug inhalation Respir. Care 47 1419-33

[5] Demirci U 2006 Acoustic picoliter droplets for emerging application in semiconductor industry and biotechnology J. Microelectromech. Syst. 15 957-66

[6] Kang T G and Cho Y-H 2005 A four-bit digital microinjector using microheater array for adjusting the ejected droplet volume J. Microelectromech. Syst. 14 1031-8

[7] Bergkvist J, Lilliehorn T, Nilsso J, Johansson S and Laurell T 2005 Miniaturized flowthrough microdispenser with piezoceramic tripod actuation J. Microelectromech. Syst. 14 134-40

[8] Naik N et al 2007 Microfluidics for generation and characterization of liquid and gaseous micro- and nanojets Sensors Actuators 134 119-27

[9] Liu J, Lin J-M and Knopp D 2008 Using a circular groove surrounded inlet to generate monodisperse droplets inside a microfluidic chip in a gravity-driven manner J. Micromech. Microeng. 18115005

[10] Teh S-Y, Lin R, Hungb L-H and Lee A P 2008 Droplet microfluidics Lab Chip 8 198-220

[11] Anna S L, Bontoux N and Stone H A 2003 Formation of dispersions using flow-focusing in microchannels Appl. Phys. Lett. 82 364-6

[12] Hsiung S-K, Chen C-T and Lee G-B 2006 Micro-droplet formation utilizing microfluidic flow focusing and controllable moving-wall chopping techniques J. Micromech. Microeng. 16 2403-10

[13] Hsiung S-K, Lee C-H and Lee G-B 2007 A tunable microflow focusing device utilizing controllable moving walls and its applications for formation of micro-droplets in liquids J. Micromech. Microeng. 17 1121-9

[14] Lee G-B, Chang C-C, Huang S-B and Yang R-J 2004 The hydrodynamic focusing effect inside rectangular microchannels J. Micromech. Microeng. 16 1024-32

[15] Chang C-C, Huang Z-X and Yang R-J 2007 Three-dimensional hydrodynamic focusing in two-layer polydimethylsiloxane (PDMS) microchannels $J$. Micromech. Microeng. 17 1479-86

[16] Huang S-H, Tan W-H, Tseng F-G and Takeuchi S 2006 A monolithically three-dimensional flow-focusing device for 
formation of single/double emulsions in closed/open microfluidic systems J. Micromech. Microeng. 16 2336-44

[17] Nisisako T and Torii T 2008 Microfluidic large-scale integration on a chip for mass production of monodisperse droplets and particles Lab Chip 8 287-93

[18] Chang F-C and Su Y-C 2008 Controlled double emulsification utilizing 3D PDMS microchannels J. Micromech. Microeng. 18065018 (8pp)

[19] Ong W-L, Hua J, Zhang B, Teo T-Y, Zhuo J, Nguyen N-T, Ranganathan N and Yobas L 2007 Experimental and computational analysis of droplet formation in a high-performance flow-focusing geometry Sensors Actuators 138 203-12

[20] Takeuchi S, Garstecki P, Weibel D B and Whitesides G M 2005 An axisymmetric flow-focusing microfluidic device Adv. Mater. 17 1067-72

[21] Lucía M-B, María F-M, Pascual R-C, Alfonso R-G, Cebolla Á, Chávez S and Gañín-Calvo A M 2005 Flowfocusing: a versatile technology to produce size-controlled and specific-morphology microparticles Small 1 688-92

[22] Luque A, Perdigones F, Esteve J, Montserrat J, Gañín-Calvo A and Quero J M 2007 Silicon microdevice for emulsion production using three-dimensional flow focusing J. Microelectromech. Syst. 16 1201-8
[23] Gañán-Calvo A M 2004 Enhanced liquid atomization: from flow focusing to flow blurring Appl. Phys. Lett 86214101

[24] Gañán-Calvo A M 1998 Generation of steady liquid microthreads and micron-sized monodisperse sprays in gas streams Phys. Rev. Lett. $80285-8$

[25] Martín-Banderas L, Rodríguez-Gil A, Cebolla Á, Chávez S, Teresa B-Á, Fernandez Garcia J M, Flores-Mosquera M and Gañán-Calvo A M 2006 Towards high-throughput production of uniformly encoded microparticles $A d v$. Mater. 18 559-64

[26] Nieuwenhuis J H, Bastemeijer J, Sarroc P M and Vellekoopa M J 2003 Integrated flow-cells for novel adjustable sheath flows Lab Chip 3 56-61

[27] Sundararajan N, Pio M S, Lee L P and Berlin A A 2004 Three-dimensional hydrodynamic focusing in polydimethylsiloxane (PDMS) microchannels J. Microelectromech. Syst. 13 559-67

[28] Gañán-Calvo A M, López-Herrera J M and Riesco-Chueca P 2006 The combination of electrospray and flow focusing J. Fluid. Mech. 566 421-45

[29] Bocanegra R, Galán D, Márquez M, Loscertales I G and Barrero A 2005 Multiple electrosprays emitted from an array of holes Aerosol Sci. 36 1387-99 